\title{
Accuracy of Needle Placement in Cadavers: Non-Guided Versus Ultrasound-Guided
}

\author{
Jae Sung Yun, MD, Min Jae Chung, MD, Hae Rim Kim, MD, Jae In So, MD, \\ Jung Eun Park, MD, Hyun Mi Oh, MD, Jong In Lee, MD \\ Department of Rehabilitation Medicine, Seoul St. Mary's Hospital, \\ The Catholic University of Korea College of Medicine, Seoul, Korea
}

\begin{abstract}
Objective To compare the accuracy rates of non-guided vs. ultrasound-guided needle placement in four lower limb muscles (tibialis posterior, peroneus longus, and short and long heads of the biceps femoris).

Methods Two electromyographers examined the four muscles in each of eight lower limbs from four fresh frozen cadavers. Each electromyographer injected an assigned dye into each targeted muscle in a lower limb twice (once without guidance, another under ultrasound guidance). Therefore, four injections were done in each muscle of one lower limb. All injections were performed by two electromyographers using 18 gauge 1.5 inch or 24 gauge 2.4 inch needles to place $0.5 \mathrm{~mL}$ of colored acryl solution into the target muscles. The third person was blinded to the injection technique and dissected the lower limbs and determined injection accuracy.

Results A 71.9\% accuracy rate was achieved by blind needle placement vs. $96.9 \%$ accuracy with ultrasoundguided needle placement ( $\mathrm{p}=0.001$ ). Blind needle placement accuracy ranged from $50 \%$ to $93.8 \%$.

Conclusion Ultrasound guidance produced superior accuracy compared with that of blind needle placement in most muscles. Clinicians should consider ultrasound guidance to optimize needle placement in these muscles, particularly the tibialis posterior.
\end{abstract}

Keywords Cadaver, Electromyography, Ultrasound, Tibialis posterior, Biceps femoris

\section{INTRODUCTION}

Nerve conduction studies and needle electromyography (EMG) are usually used to diagnose neuromuscular

Received July 30, 2014; Accepted October 8, 2014

Corresponding author: Jong In Lee

Department of Rehabilitation Medicine, Seoul St. Mary's Hospital, The Catholic University of Korea College of Medicine, 222 Banpo-daero, Seocho-gu, Seoul 137-701, Korea

Tel: +82-2-2258-2822, Fax: +82-2-2258-2825, E-mail: ljikyh@catholic.ac.kr

(c) This is an open-access article distributed under the terms of the Creative Commons Attribution Non-Commercial License (http://creativecommons. org/licenses/by-nc/3.0) which permits unrestricted noncommercial use, distribution, and reproduction in any medium, provided the original work is properly cited.

Copyright $\odot 2015$ by Korean Academy of Rehabilitation Medicine disease. Anatomical landmarks and auditory feedback by selectively activating muscles are used to localize a muscle during needle EMG. However, if unfamiliar muscles are tested or there are adjacent muscles showing a similar activation pattern but are innervated by a different peripheral nerve or root, it may be difficult to accurately place the needle [1]. Additionally, it is impossible to accurately place a needle in some situations, such as when anatomical landmarks disappear due to surgery or trauma or when auditory feedback is unavailable due to the absence of voluntary muscle contraction in an unconscious patient [1]. Inaccurate needle placement can cause poor diagnostic utility of EMG and damage nerves, 
vessels, and organs $[1,2]$.

The use of ultrasonography (US) has been increasing because of its advantages, such as no radiation exposure, quick scan time, and utility for observing soft tissue [3]. Previous studies have reported that the accuracy rate of needle EMG using anatomical landmarks is low $[1,2]$. Therefore, needle EMG using US has been studied recently $[1,4]$.

US makes it possible to evaluate anatomical landmarks, fascial planes, and the neurovascular structure of the intended target by providing direct and real-time visualization of soft tissue, thereby enhancing safety and accuracy during needle placement $[4,5]$. Although lower limb muscles are commonly selected for a needle EMG examination, US-guided needle placement in lower limb muscles has been studied little, and sample size was quite small [1]. Furthermore, the study of Boon et al. [1] was performed using disarticulated lower limbs, which made it impossible to palpate proximal landmarks located in the pelvis or hip. Moreover, it may be difficult to maintain constant limb position when placing a needle in a disarticulated lower limb.

Therefore, we compared the accuracy rates of US-guided needle placement vs. blind needle placement using superficial landmarks in lower limb muscles articulated with the pelvic girdle, which are usually used for a needle EMG examination, via cadaver dissection.

\section{MATERIALS AND METHODS}

\section{Subjects}

Four muscles (short and long heads of the biceps femoris, tibialis posterior, and peroneus longus) in each of eight lower limbs from four fresh-frozen cadavers (three men and one woman; mean age, 67.5 years) were examined. No significant deformities, trauma, or changes due to lower extremity surgery were found in the four cadavers.

\section{Methods}

Two electromyographers examined the four muscles in each of the eight cadaver lower limbs using an identical needle EMG examination (blind needle placement based on superficial landmarks) and US-guided needle placement, respectively. In most cases, an 18 gauge 1.5 inch needle was inserted, except for the tibialis posterior muscle, in which a 24 gauge 2.4 inch needle was used. Two electromyographers participated in this study. The first was a rehabilitation physician and an experienced electromyographer and sonographer. The second was a rehabilitation resident who had 1 year of clinical experience with EMG; however, he was a US novice. Each electromyographer injected different colored dyes. The rehabilitation physician used a red dye for the blind injections and a blue dye for the US-guided injections. The rehabilitation resident used a yellow dye for the blind injections and a black dye for the US-guided injections.

Blind needle placement based on superficial landmarks The two physicians injected dye into the targeted muscle in each of the eight lower limbs twice. The first injection was blind and based on superficial landmarks, whereas the second was done under ultrasound guidance. Thus, the four targeted muscles in a single lower limb were tested twice by each electromyographer, as described above. Therefore, each lower limb was needled eight times by a physician. Thus, 64 dye injections were performed by each physician for the eight lower limbs. Accordingly, the two physicians performed 128 dye injections.

Acrylic gel and oil-based paints were mixed to increase viscosity, and $0.5 \mathrm{~mL}$ of this colored acryl solution was injected into each targeted muscle. The needle was inserted, and dye (red or yellow) was injected as described below after localizing each muscle according to Perotto and Delagi [6].

\section{Tibialis posterior}

A posterior medial approach was used to insert the needle from the tibial tuberosity at one handbreath distal and one fingerbreath (FB) medial. The needle was placed through the flexor digitorum longus and the soleus immediately behind the tibia.

\section{Peroneus longus}

This muscle originates in the head and proximal twothirds of the fibula and inserts into the first metatarsal and cuneiform. The injection was made at a point three FB distal to the fibular head.

\section{Short head of the biceps femoris}

The linea aspera is the origin, and it inserts into the 
fibular head and lateral condyle of the tibia. The needle was inserted four FB proximal to the fibular head, and the long head of the biceps was palpated where it passed through the medial side.

\section{Long head of the biceps femoris}

The origin is the ischial tuberosity, and insertion is at the fibular head. The needle was placed at the midpoint of a line between the fibular head and ischial tuberosity.

\section{Needle placement under ultrasound guidance}

Each muscle was identified, and the dye (blue or black) injection was completed with visualization of the needle and its tip under US guidance. The needle was placed into the tibialis posterior and peroneus longus in the supine position, whereas it was placed into the short and long heads of the biceps femoris in the prone position.

An anterior approach was used to inject dye into the tibialis posterior muscle under US guidance, whereas a posterior medial approach was used for blind needle placement (Fig. 1).

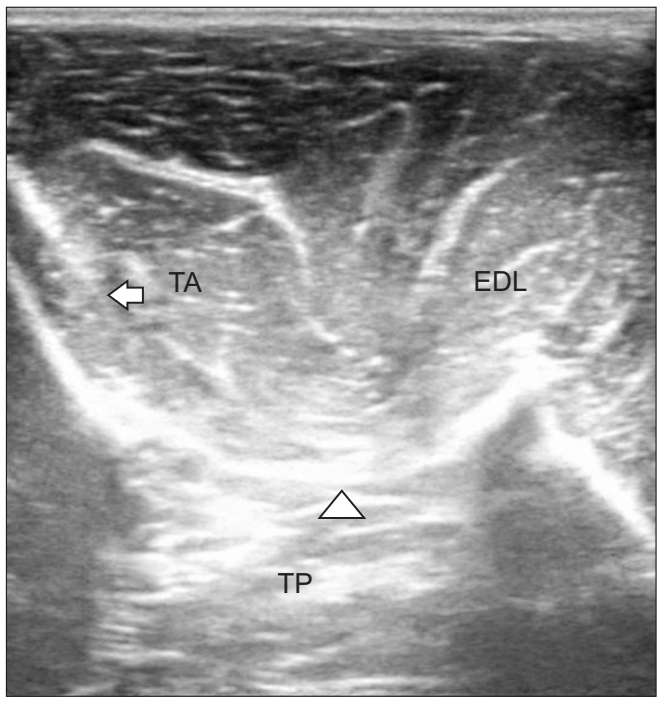

Fig. 1. The tibialis anterior (TA), extensor digitorum longus (EDL), and tibialis posterior (TP) are presented on an ultrasound-guided needle placement image (transverse) of the tibialis posterior. Needle (arrow) passed through the TA and interosseous membrane (arrow head), and injectate placed into the tibialis posterior.

Table 1. Accuracy of blind and ultrasound-guided dye injection

\begin{tabular}{lcccc}
\hline \multirow{2}{*}{ Muscle } & \multicolumn{2}{c}{ Non-guided } & \multicolumn{2}{c}{ Ultrasound-guided } \\
\cline { 2 - 5 } & Correct placement & Accuracy (\%) & Correct placement & Accuracy (\%) \\
\hline Peroneus longus & & & & \\
EM 1 & $6(8)$ & 75.00 & $8(8)$ & 100 \\
EM 2 & $6(8)$ & 75.00 & $8(8)$ & 100 \\
Total (p=0.033) & $12(16)$ & 75.00 & $16(16)$ & 100 \\
Tibialis posterior & & & & \\
EM 1 & $4(8)$ & 50.00 & $8(8)$ & 100 \\
\hline EM 2 & $4(8)$ & 50.00 & $8(8)$ & 100 \\
Total (p=0.001) & $8(16)$ & 50.00 & $16(16)$ & 100 \\
Long head of biceps femoris & & & & \\
EM 1 & $7(8)$ & 87.50 & $8(8)$ & 100 \\
EM 2 & $8(8)$ & 100 & $7(8)$ & 97.50 \\
Total (p=1.000) & $15(16)$ & 93.75 & $15(16)$ & 93.75 \\
Short head of biceps femoris & & & & 100 \\
EM 1 & $6(8)$ & 75.00 & $8(8)$ & 87.50 \\
\hline EM 2 & $5(8)$ & 62.50 & $7(8)$ & 93.75 \\
Total (p=0.070) & $11(16)$ & 68.75 & $15(16)$ & 96.87 \\
\hline Overall accuracy (p=0.001) & $48(64)$ & 71.87 & $62(64)$ & \\
\hline
\end{tabular}

Numbers in parentheses indicate number of attempts.

EM 1, electromyographer 1 (a rehabilitation resident with 1 year of clinical experience in electromyography); EM 2, electromyographer 2 (a rehabilitation physician and experienced electromyographer).

p-value between the accuracy of non-guided and ultrasound-guided injections. 
Table 2. Error locations for blind and ultrasound-guided needle placement

\begin{tabular}{lll}
\hline \multicolumn{1}{c}{ Muscle } & \multicolumn{1}{c}{ Error location } \\
\cline { 2 - 3 } & \multicolumn{1}{c}{ Non-guided } & Ultrasound-guided \\
\hline Peroneus longus & $\begin{array}{l}\text { Tibialis anterior (2) } \\
\text { Extensor digitorum longus (2) }\end{array}$ & Semimembranosus (1) \\
Tibialis posterior & Soleus (8) & Subcutaneous fat (1) \\
\hline Long head of biceps femoris & Semimembranosus (1) \\
\hline Short head of biceps femoris & Subcutaneous fat (4) \\
\hline
\end{tabular}

Numbers in parentheses indicate how many times the injectate was placed incorrectly.

The Accuvix XG portable US system (Medison, Seoul, Korea) and a 3-12 MHz linear array transducer (Medison) were used. The skin-transducer interface was coupled acoustically using a standard US gel.

\section{Evaluation}

Excluding the two electromyographers who performed the dye injections, three physicians, who were blinded to the injection method, dissected cadavers as co-investigators and determined whether the injectate was delivered accurately to each targeted muscle. Needle placement was graded as 'accurate' if the injectate was located exactly in the targeted muscle or as 'inaccurate' if it was not in the targeted area.

\section{Statistical analysis}

Data were analyzed using SPSS ver. 18.0 for Windows (SPSS Inc., Chicago, IL, USA). The chi-square test was used to analyze differences between the accuracy rates of the blind and US-guided needle placements and differences in the accuracy rates between electromyographers. p-values less than 0.05 were considered significant.

\section{RESULTS}

\section{Blind vs. US-guided needle placement}

The overall accuracy rates of blind and US-guided needle placement were $71.9 \%$ and $96.9 \%$, respectively $(\mathrm{p}=$ 0.001 ) (Table 1). The accuracy rate of blind needle placement varied according to muscle type, ranging from $50 \%$ to $93.8 \%$. Accuracy was lowest in the tibialis posterior (Table 1). Blind needle insertion into the tibialis posterior was attempted 16 times and failed eight times. All incorrect injectates were placed in the soleus (Table 2). The accuracy rate of US-guided needle placement was

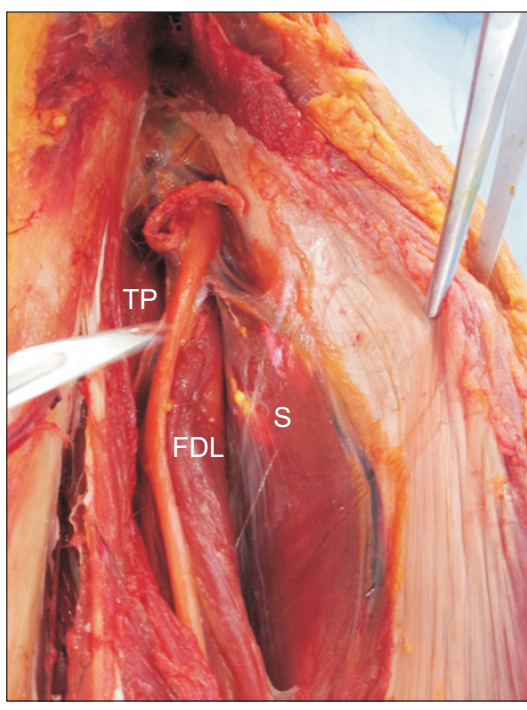

Fig. 2. Photograph of a dissected cadaver with incorrectly placed injectate (yellow and red) into the soleus. The needle passed under the intended tibialis posterior target and into the soleus. TP, tibialis posterior; FDL, flexor digitorum longus; $\mathrm{S}$, soleus.

$93.75 \%-100 \%$, depending on the specific muscle examined.

Fig. 2 is a photograph of a dissected cadaver after blind and US-guided injections. It shows that the needle passed under the intended tibialis posterior target, and that injectate (yellow or red) was placed inaccurately into the soleus instead of the tibialis posterior (Fig. 2).

\section{Comparison between the two electromyographers}

No significant differences in the accuracy rates of blind or US-guided needle placement were observed between the two electromyographers $(p=1.000$ and $p=0.151$, respectively). 


\section{DISCUSSION}

Inappropriate needle placement can compromise the diagnostic and therapeutic utility of a procedure. Prior research has indicated the potential for incorrect needle placement without imaging guidance. In a study by Haig et al. [2], the accuracy rates of blind needle placement into the long and short heads of the biceps femoris, tibialis posterior, and peroneus longus were $70 \%, 30 \%, 10 \%$, and $50 \%$, respectively. Boon et al. [1] evaluated the same muscles with accuracy rates of $25 \%, 100 \%, 50 \%$, and $75 \%$, respectively. The accuracy rates of blind needle placement varied by muscle because the number of injections was small, such as 10 and four times, and there may have been differences in clinical experience between the electromyographers $[1,2]$. Furthermore, the accuracy of blind needle placement into the long head of the biceps femoris was as low as $25 \%$ in the study of Boon et al. [1]. That study was performed using disarticulated lower limbs; thus, it would have been impossible to palpate the ischial tuberosity as a proximal landmark.

Considering these factors, 16 needle insertions were done for each muscle in our study, using blind and USguided needles. We examined the accuracy rates of blind vs. US-guided needle placement in the long and short heads of the biceps femoris, the tibialis posterior, and the peroneus longus muscles in eight lower limbs articulated with the pelvic girdle.

We selected these muscles because the long and short heads of the biceps femoris are innervated by the tibial or peroneal portions of the sciatic nerve. Thus, they play a key role in determining division of a sciatic nerve injury. In particular, the short head of the biceps femoris is the only peroneal-innervated muscle above the level of the fibular neck and has special importance during EMG evaluations of peroneal palsy, sciatic neuropathy, and other proximal lesions [7].

The tibialis posterior is one of the most commonly examined muscles and plays an important role when distinguishing a common peroneal nerve injury from a sciatic nerve injury, lumbosacral plexopathy, or L5 radiculopathy [7]. However, we assumed that the accuracy of needle placement in this muscle would be low because it is located deep in the posterior compartment and is difficult to palpate. As other studies have reported, the accuracy rate into this muscle is as low as $50 \%$ and $10 \%$

\section{$[1,2]$.}

The peroneus longus is the most accessible muscle innervated mainly by L 5 and the superficial peroneal nerve, and it is usually examined to detect a peroneal nerve injury. Clinicians should be careful when inserting a needle into this muscle because the needle may be placed in the soleus, extensor digitorum longus, or fibular nerve instead $[2,6]$.

US-guided needle placements into most muscles were more accurate in our study than blind needle placements (Table 1). The accuracy rate of US-guided needle placement into the short and long heads of the biceps femoris muscle was $93.8 \%$, and only one failure was observed in 16 attempts. Needles that were intended to be placed into the short head of the biceps femoris were occasionally inserted superficially, and dye was injected into subcutaneous fat. This may have been due to misdiagnose of fat as muscle. In addition, attempts to place a needle into the long head of the biceps femoris occasionally passed through the muscle, and dye was misplaced in the semitendinosus muscle, which is an adjacent muscle. This may have been caused by inexperience imaging these muscles, as the two muscles are adjacent.

In this study, blind needle placement into the long head of the biceps femoris was done at the midpoint of a line between the fibular head and the ischial tuberosity. Accuracy was very high $(93.8 \%)$ when compared with that in Boon's study (25\%). As our study was performed using lower limbs articulated with the pelvic girdle, it was possible to accurately palpate pelvic landmarks. As a result, the accuracy rate of blind needle placement was lowest $(50 \%)$ in the tibialis posterior, compared with that of the other muscles, and all failed injections reached the soleus. Both the tibialis posterior and soleus are dominated by the tibial nerve. However, it is clinically critical to identify these two adjacent muscles during EMG because the tibialis posterior is innervated by L 5 and S1, whereas the soleus is innervated by L5, S1, and the S2 root. In addition, the soleus provides ankle plantar flexion with the knee flexed, whereas the tibialis posterior is involved in ankle plantar flexion and inversion. Therefore, it is also important to identify these two adjacent muscles during Botox injections for spasticity and movement disorders.

The injectates were misplaced twice into the tibialis anterior and extensor digitorum longus (EDL) muscles during blind needle placement into the peroneus longus, 
respectively. This result occurred because the needle was inserted too deep and passed through the peroneus longus and anterior portion of the fibular bone into the other muscles. The peroneus longus is dominated by the superficial peroneal nerve and is innervated by L5 and the S1 root, whereas the tibialis anterior and EDL are dominated by the deep peroneal nerve and innervated by L4 and the L5 root. Accordingly, it is clinically important to distinguish these two muscles during an EMG examination.

We found no difference in the accuracy rates between the electromyographers for either blind or US-guided needle placement. In addition, no differences in accuracy rates were observed between the electromyographers during US-guided needle placement into the individual muscles.

We found that a US novice could use US for needle placement without difficulty. Schnitzler et al. [8] studied the accuracy of blind needle placement into the gastrocnemius muscle and reported no difference in accuracy rate between naive and experienced groups, as reported in the present study.

There were several limitations of this current study. First, sample size was small due to the high cost of cadavers. Secondly, auditory feedback using selective muscle activation and firing motor unit potentials were not available for blind needle placement because our subjects were cadavers. Additionally, verifying selective muscle movement by electrical stimulation was not possible. Thus, we assume that the accuracy of EMG needle injections without US guidance may be higher in live patients.

However, there are patients who are incapable of selective muscle contraction due to cognitive impairment, postoperative anatomical variations, spasticity, or weakness. Therefore, the accuracy rates of blind needle placement determined in this study are significant considering these clinical situations.

Lastly, different approaches were used when a needle was placed into the tibialis posterior muscle with or without US guidance; a posterior medial approach was used with blind needle placement and an anterior approach was used during US-guided needle placement, respectively.

According to previous studies, the safety window, which is the distance between the tibia and the neurovascular bundle, is larger if the anterior approach is used, com- pared with that of the posterior medial approach. In particular, the upper one-third of the tibia has a wider safety window than the midpoint or the lower one-third [9-11].

Thus, needles were placed using the anterior approach at the upper one-third of tibia during US-guided tibialis posterior muscle injections in this study.

In conclusion, US has many advantages; it is painless, readily available in most outpatient departments, and does not expose the patient to radiation. Compared with blind needle placement, US guidance improved needle placement accuracy during EMG. Therefore, clinicians should consider US guidance to optimize needle placement into these muscles, particularly the tibialis posterior, which showed low accuracy during blind needle placement.

However, the electromyographer should use anatomical landmarks, voluntary muscle activation, and electrical stimulation techniques for accurate muscle targeting when US is unavailable.

\section{CONFLICT OF INTEREST}

No potential conflict of interest relevant to this article was reported.

\section{REFERENCES}

1. Boon AJ, Oney-Marlow TM, Murthy NS, Harper CM, McNamara TR, Smith J. Accuracy of electromyography needle placement in cadavers: non-guided vs. ultrasound guided. Muscle Nerve 2011;44:45-9.

2. Haig AJ, Goodmurphy CW, Harris AR, Ruiz AP, Etemad J. The accuracy of needle placement in lowerlimb muscles: a blinded study. Arch Phys Med Rehabil 2003;84:877-82.

3. Cho KH, Gee SJ, Lee HJ, Hwang SH. Comparision of blind technique and ultrasonography guided technique of subacromial subdeltoid bursa injection. J Korean Acad Rehabil Med 2010;34:209-13.

4. Smith J, Hurdle MF, Locketz AJ, Wisniewski SJ. Ultrasound-guided piriformis injection: technique description and verification. Arch Phys Med Rehabil 2006;87:1664-7.

5. Boon AJ, Alsharif KI, Harper CM, Smith J. Ultrasoundguided needle EMG of the diaphragm: technique description and case report. Muscle Nerve 2008;38:1623-6. 
6. Perotto AO, Delagi EF. Anatomical guide for the electromyographer: the limbs and trunk. 4th ed. Springfield: Charles C Thomas; 2005.

7. Preston DC, Shapiro BE. Electromyography and neuromuscular disorders: clinical-electrophysiologic correlations. 2nd ed. Philadelphia: Butterworth-Heinemann; 2005.

8. Schnitzler A, Roche N, Denormandie P, Lautridou C, Parratte B, Genet F. Manual needle placement: accuracy of botulinum toxin A injections. Muscle Nerve 2012;46:531-4.

9. Rha DW, Im SH, Lee SC, Kim SK. Needle insertion into the tibialis posterior: ultrasonographic evaluation of an anterior approach. Arch Phys Med Rehabil 2010;91:283-7.

10. Semple R, Murley GS, Woodburn J, Turner DE. Tibialis posterior in health and disease: a review of structure and function with specific reference to electromyographic studies. J Foot Ankle Res 2009;2:24.

11. Yang SN, Lee SH, Kwon HK. Needle electrode insertion into the tibialis posterior: a comparison of the anterior and posterior approaches. Arch Phys Med Rehabil 2008;89:1816-8. 